ESAIM: PROCEEDINGS, September 2005, Vol.14, 25-40

Eric Cancès \& Jean-Frédéric Gerbeau, Editors

DOI: $10.1051 /$ proc:2005003

\title{
OSCILLATORY FLOW IN A TUBE WITH TIME-DEPENDENT WALL DEFORMATION AND ITS APPLICATION TO MYOCARDIAL BRIDGES*
}

\author{
Bernhard, S. ${ }^{1}$, Möhlenkamp, S. ${ }^{2}$, Erbel, R. ${ }^{2}$ And Tilgner, A. ${ }^{1}$
}

\begin{abstract}
In this paper we numerically investigate a one-dimensional model of blood flow in the human coronary arteries. The nonlinear hyperbolic system is expressed in terms of the cross-sectional area, flow velocity and pressure $(\mathrm{A}, \mathrm{u}, \mathrm{p})$. The more widely studied linearised system is also discussed where conservation of static pressure, instead of total pressure, is enforced. The method of outgoing characteristics is used to satisfy the interface conditions, while a three-element windkessel model is adopted as outflow condition at the terminals of the network. Inside the segmental domain the leap-frog method is used for numerical integration. Within the context of this model we pay particular attention to the case when abrupt or smooth, space and time dependent variation of cross-sectional area of an artery is caused by externally prescribed motion of the vessel walls (e.g. myocardial bridge, flow watch). The derivation of the model and the numerical implementation are detailed. They are applied to model numerical experiments of the arterial system. Additionally to a system studied in $[10,15,22,28]$ the coronary arteries are parameterised. The main features of the flow through myocardial bridges are discussed.
\end{abstract}

\section{INTRODUCTION}

The science of understanding the processes occurring in human arterial system reaches back to the $16^{\text {th }}$ century, where Harvey proposed pulsatile blood flow is based on the periodic blood ejection of the heart combined with a continuous flow. A simplified one-dimensional description of the human arterial system was introduced by Euler in 1775 who derived a system of nonlinear partial differential equations, expressing the conservation of mass and momentum for inviscid flow. However, the wave nature of the arterial flow was first mentioned by Young who derived the constitutive equations describing the behaviour of the elastic wall with changes in transmural pressure. In 1877 Moens and Kortweg independently found a relation for the wave speed of pressure-flow waves in thin-walled elastic tubes. Today the equation is known as the Moens-Kortweg equation for the wave speed. The two-dimensional equations for flow in straight, circular elastic tube were linearised by Womersley in 1957 and he obtained a wave solution by the assumption of linear superposition of harmonic waves. Yet in 1860 Riemann provided the analytical tools for the system of hyperbolic equations when he introduced the method of characteristics. An introduction to this method can be found in $[6,26]$. Due to the fact that physiological conditions of the human arterial system are only weakly nonlinear, many characteristics of the flow can be captured by the linearised system. In spite of many simplifications made

* The work of this project was partially funded by a Georg-Christoph-Lichtenberg Scholarship.

1 University of Göttingen, Department of Physics, Friedrich-Hund-Platz 1,

37077 Göttingen, Germany; e-mail: Stefan.Bernhard@Physik.Uni-Goettingen.de

2 University Clinic of Essen, West-German Heart Center, Clinic of Cardiology, (Director: Prof. Dr. R. Erbel),

Hufelandstrasse 55, 45122 Essen, Germany; e-mail: stefan.moehlenkamp@uni-essen.de 
by the one-dimensional description of wave propagation, they are excellent applicable to evaluate the dynamic behaviour of blood circulation and to obtain boundary conditions. An overview reporting the concepts and results are given in $[2,5,12,18,21,23,35]$.

The objective of this paper is to review the one-dimensional model starting from first principles and to demonstrate how these equations can be applied to linear and nonlinear numerical modelling of externally enforced wall deformation with space and time. An important clinical example for external wall deformation is myocardial bridging, a normal variant characterised by compression of the coronary arteries due to myocardial muscle fibres overlying a segment of the artery. They are most commonly found in the middle segment of the left anterior descending coronary artery (LAD), at a depth of 1 to $10 \mathrm{~mm}$ with a typical length of 10 to $30 \mathrm{~mm}$ [17].

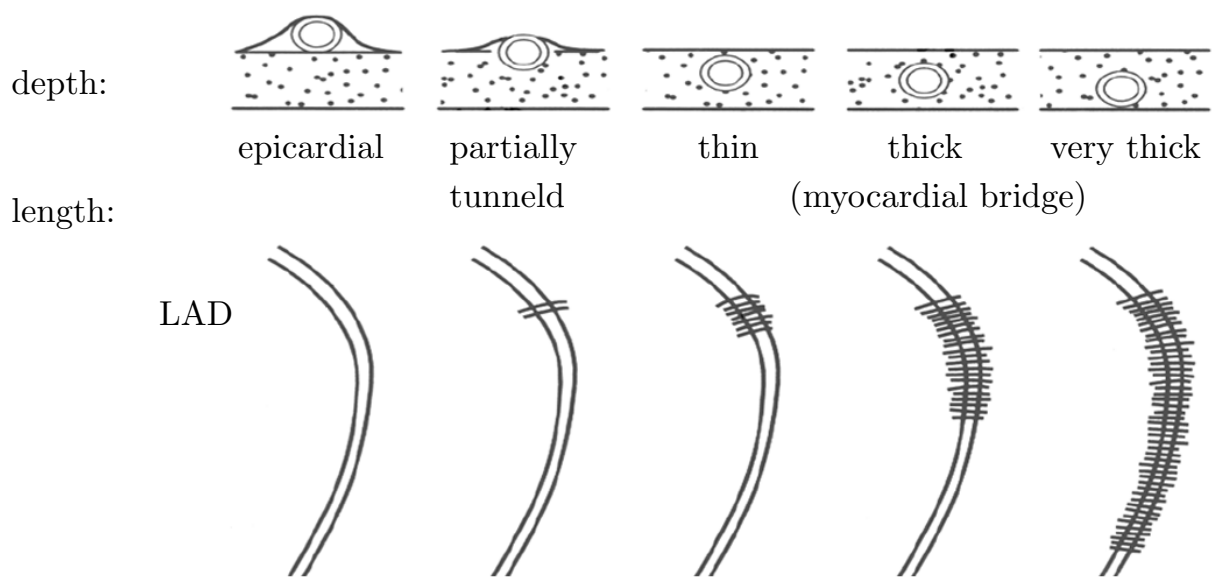

FiguRE 1. Schematic illustration of myocardial bridges of different length and depth in the mid LAD, modified from B.F. Waller, Hurst's The Heart, 9. Edition chapter 42.

The paper is organised as follows, in section 1.1 we first state our pathological motivation for modelling time and space depending wall motion. After simplification of the muscle bridge anatomy in section 1.2, we introduce the concept of a sectional algebraic pressure-area relationship for different degrees of deformation in 1.3. The governing equations for conservation of mass and momentum in a single one-dimensional vessel are reviewed and additional terms in the system are discussed in section 1.4. We subsequently construct both the linear and nonlinear systems in terms of characteristic variables to satisfy the interface and boundary conditions. In section 2.2 we extend the single vessel formulation to a network by modelling the interfaces including both bifurcations and simple connections of vessel segments using Riemann invariants and interface conditions for the pressure and flow. Having introduced the parameters of the network, we complete the description by applying boundary conditions at the outflow, which are enforced using a three-element windkessel model causing the outgoing wave to be partially reflected back into the system. For numerical discretisation of the governing (A, $\mathrm{u}$, p) system we use a continuous leap-frog formulation with a one-dimensional spatial approximation. The leap-frog method is commonly known as fast convergent with good dispersion properties. Finally in section 3 we apply the one-dimensional model to the human arterial network including the major 55 arteries, previously studied in $[10,15,22,28]$, additionally the major 48 coronary arteries are parameterised. In section 4 we analyse secondary flow effects during external deformation of the wall and the influence on the flow and pressure waveforms in a model system of the left coronary arteries (LCA). To evaluate the effects of the myocardial bridge we investigate the system for low, normal and high peripheral resistance. 


\section{Problem Formulation}

\subsection{Pathological Condition}

Under normal circumstances, coronary arteries have diameters large enough to transport sufficient amounts of oxygen to myocardial cells. Increases in myocardial oxygen demand, e.g. during exercise, are met by increases in coronary artery blood flow because - unlike in many other organs - extraction of oxygen from blood cannot be increased. This is in part mediated by increases in diameters of small intra myocardial arteries. Yet, the large proximal (epicardial) coronary arteries contribute only a small fraction of total vascular resistance and show little variation in diameter during the cardiac cycle at any given metabolic steady state.

The most common cause of an impaired ability to match oxygen supply and demand is coronary atherosclerosis, a disease that eventually leads to fixed coronary artery lumen narrowing, impaired coronary blood flow and potentially myocardial infarction. However, some people present with chest pain caused by phasic lumen obstruction due to myocardial bridging, were first mentioned by Reyman in 1737 [25]. In this anatomic variant, a coronary artery segment courses underneath myocardial fibres resulting in vessel compression during systole, i.e. the myocardial contraction phase [17]. An angiogram of two myocardial bridges in series shown in figure 2 (a). Although coronary blood flow occurs predominantly during diastole, i.e. the filling phase of the hearts chambers, total blood flow may nonetheless be reduced partly because vascular relaxation may extend significantly into diastole, the myocardial relaxation phase. Within the bridged segments permanent diameter reductions of $22-58 \%$ were found during diastole, while in systole the diameters were reduced by $70-95 \%$ [14]. A schematic drawing of the increased flow velocities $(\mathrm{cm} / \mathrm{s})$ during systole $(31.5$ within versus 17.3 proximal and 15.2 distal) is given in figure 2 (b).

(a)
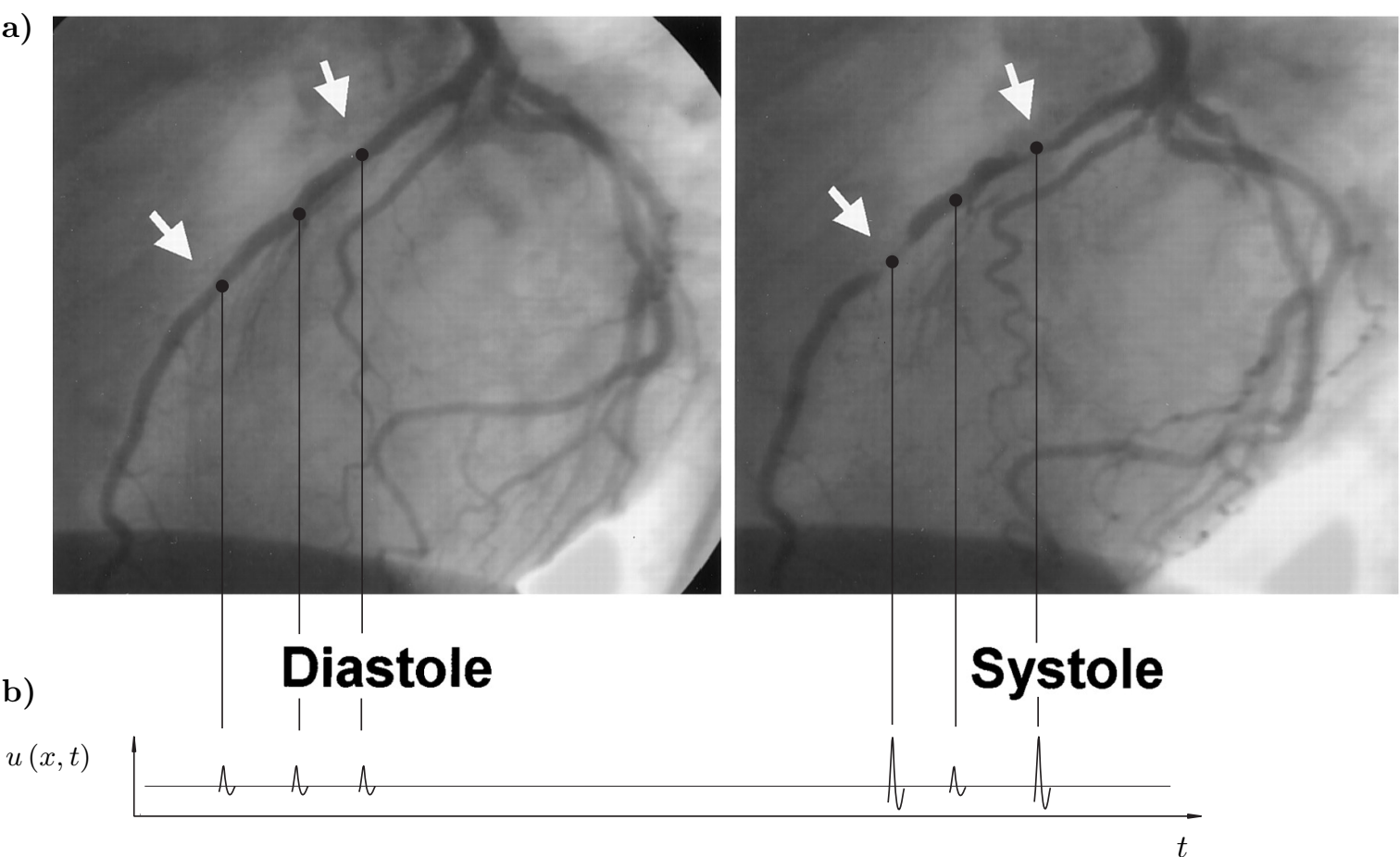

FiguRE 2. (a) Coronary angiogram of two myocardial bridges in the left anterior descending (LAD) branch (arrows) in diastole (left) and systole (right). Compression of the artery during the hearts contraction phase, i.e. systole, is a characteristic finding in myocardial bridging (see text and [17] for details). (b) Diastolic lumen dimensions and flow velocity are normal, while systolic flow velocities are increased within the bridged segments. 
In summary myocardial bridges can be characterised as phasic systolic vessel compression with a persistent diastolic diameter reduction, increased blood flow velocities, retrograde flow, and a reduced flow reserve. The underlying mechanisms are three fold. Firstly the discontinuity causes wave reflections, secondly the dynamic reduction of the vessel diameter produces secondary flow and thirdly there is evidence for vertebration in transition regions $[4,5,12,23]$. We primarily substantiate the increased flow velocities, the secondary flow and pressure gradient in myocardial bridges.

\subsection{Deformation Topology}

Theories of longitudinal waves in tubes, with or without nonuniformities, non-linearity and frictional dissipation, are based on the idea that variation of excess pressure $p_{e}=p_{\text {int }}-p_{\text {ext }}$ over a cross-section is negligible. The internal and external pressure of the artery at a given position $x$ at time $t$ are given by $p_{\text {int }}(x, t)$ and $p_{\text {ext }}(x, t)$ respectively. Henceforth we assume the external pressure to be zero so that $p(x, t)=p_{e}=p_{\text {int }}$ and consequently it is the excess pressure whose gradients produce fluid acceleration.

Our first simplification for modelling the blood flow in arteries is that the curvature of the tube is assumed to be small everywhere and that the flow direction in the cardiovascular system is unidirectional, so that the problem can be defined in one space dimension along the $x$-axis. According to this we have simplified the anatomy of the myocardial bridge as shown in figure 3.

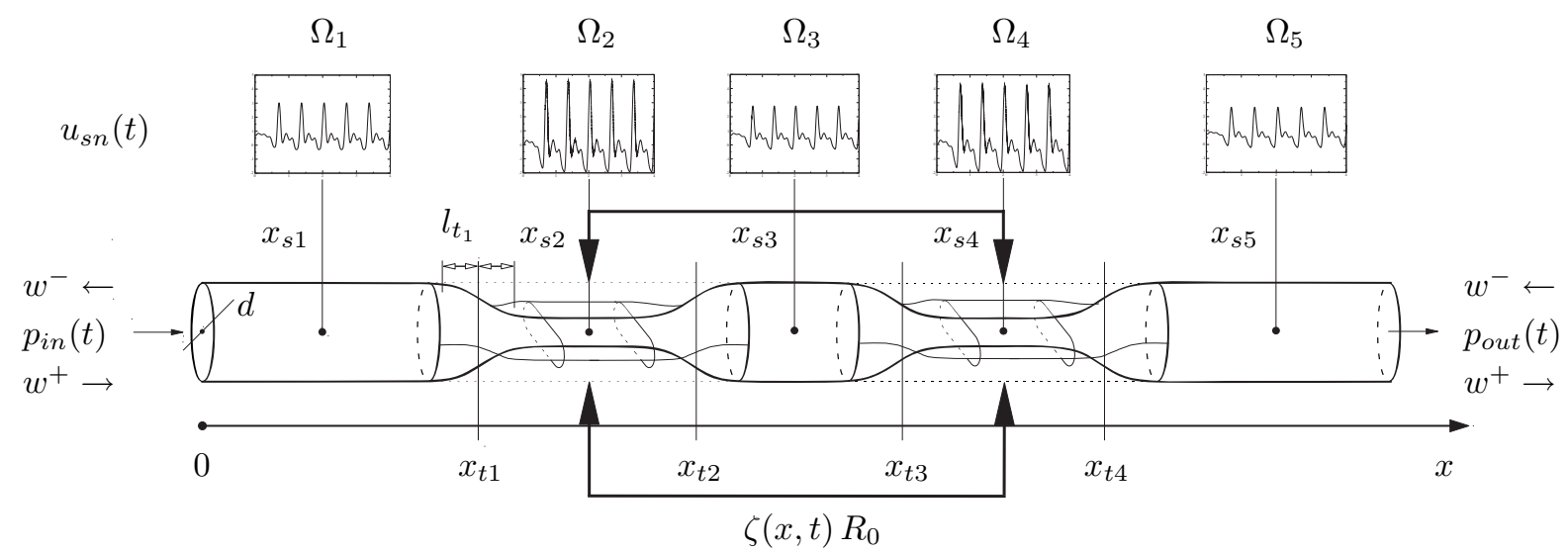

FIGURE 3. Schematic anatomy of a double myocardial bridge. The control segments $\Omega_{n}$ are equally spaced. Observation locations for hemodynamic properties are given in the center of each segment and by $x_{s n}$, transitions between the segments are at $x_{t n}$.

The two sets of anti-parallel, vertical arrows in figure 3 indicate the location of external deformation. Due to the fact that the wall thickness $h_{0}$ is small compared to the bending radius $R_{d}$, we assume that the bending stress inside the wall is negligible. Under these circumstances the deformation of the tube in $z$-direction forms a rectangle with two semi-circles as illustrated in figure 4. This is consistent with the predominately eccentric deformation of bridged segments found in [1]. The plate distance and the flat portion are denoted by $D(x, t)$

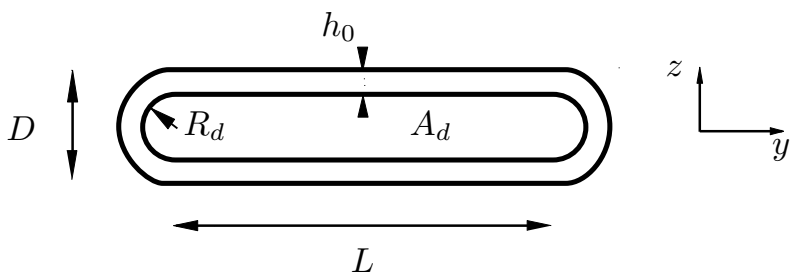

Figure 4. The cross-section $\mathrm{S}$ of a linear elastic tube under parallel deformation along the $z$-axis. We have assumed negligible bending stress inside the wall. 
and $L(x, t)$ respectively. $R_{0}$ is the inner radius and $U_{0}=2 \pi R_{0}$ is the circumference of the cylindrical tube at zero excess pressure. The equilibrium cross-sectional area of the deformed tube is $A_{d}(x, t)$, while the total cross-sectional area in the $y z$-plane of the tube is defined by $A(x, t)=\int_{S} d \sigma$. Consequently the average velocity $u(x, t)=\frac{1}{A} \int_{S} \hat{u} d \sigma$ and the excess pressure $p(x, t)=\frac{1}{A} \int_{S} \hat{p} d \sigma ; \hat{u}$ and $\hat{p}$ are the values of flow velocity and pressure in $S$. The volume flux across a given section therefore is $Q(x, t)=A u$. The curvature of deformation along the tube is characterised by $\zeta(x, t)$, which for $N$ myocardial bridges in series is chosen as

$$
\zeta(x, t)=1-\zeta_{0} f(t) \sum_{n=1}^{N}\left[\tanh \left(\frac{x-x_{t_{n}}}{l_{t_{n}}}\right)-\tanh \left(\frac{x-x_{t_{n+1}}}{l_{t_{n+1}}}\right)\right],
$$

where $\zeta_{0}$ is the degree of deformation and number between 0 and 1 , and $f(t)$ is a periodic function dependent on the contraction of the muscle fibres overlaying the artery. The transition length $l_{t_{n}}$ alters the steepness of curvature between the segmental domains $\Omega_{n}$.

\subsection{Pressure-Area relationship}

In the following we restrict our attention to an algebraic pressure-area relationship and the distensibility of the wall. The cross-sectional area is varying with the local excess pressure and the degree of deformation according to $A=A(\zeta, p)$. If we assume that $A^{\prime}$ is the perturbation about the equilibrium area $A_{d}$ the total cross-sectional area can be written as $A(\zeta, p)=A^{\prime}(\zeta, p)+A_{d}(\zeta)$. Further we assume that the deformation is symmetric about the $x y$-plane and small compared to the body dimensions. For a homogeneous, thin-walled $\left(h_{0} / R_{0} \ll 1\right)$, linear elastic tube tethered along the $x$-axis the external forces are reduced to stresses acting in the circumferential and longitudinal direction. From what is often known as Laplace's law we get the tensile stress $\tau$ per unit length of the tube, which causes circumferential elongation $\triangle U$ of the shell by $\epsilon=\frac{\Delta U}{U_{0}}$. Therefore

$$
\tau=\frac{R_{d} p}{h_{0}}\left(1-\sigma^{2}\right)=\epsilon E
$$

where $E(x, t)=E_{\theta}=E_{x}$ is the elastic modulus and $\sigma=\sigma_{\theta}=\sigma_{x}$ is the Poisson ratio in the circumferential and longitudinal direction respectively. Since biological tissue is practically incompressible $\sigma \approx \frac{1}{2}$. The equilibrium condition is obtained by balancing the forces resulting from circumferential and longitudinal tensile stress and the excess pressure, so that

$$
p(x, t)=\frac{E h_{0}}{\left(1-\sigma^{2}\right)} \frac{\triangle U}{\zeta R_{0} U_{0}}=\frac{E h_{0}}{\left(1-\sigma^{2}\right)} \frac{2 \pi \zeta R_{0}+2 L(\zeta, p)-U_{0}}{\zeta R_{0} U_{0}} .
$$

By rearranging we obtain

$$
L(\zeta, p)=\frac{1}{2}\left[\frac{1-\sigma^{2}}{E h_{0}} \zeta R_{0} U_{0} p+U_{0}-2 \pi \zeta R_{0}\right] .
$$

According to this, the pressure and deformation dependent cross-sectional area is

$$
A(\zeta, p)=\pi\left(\zeta R_{0}\right)^{2}+2 L(\zeta, p) \zeta R_{0}
$$

whereas the deformation dependent equilibrium area is

$$
A_{d}(\zeta)=\zeta R_{0} U_{0}-\pi\left(\zeta R_{0}\right)^{2}
$$

and finally

$$
A^{\prime}(\zeta, p)=\left(\zeta R_{0}\right)^{2} U_{0} p \frac{1-\sigma^{2}}{E h_{0}}
$$

is the pressure induced perturbation. It should be noted that under the assumption of linear elastic material with constant elastic modulus, equation (5) and (7) have the property that the area increases linearly with 
excess pressure. Real arteries however resist over-expansion by having a incremental Young's modulus that increases with increasing strain [35]. Moreover the area perturbation in equation (7) is not only dependent on pressure variation but also on the degree of deformation. This is due to the fact that the distensibility of the tube depends in some way on the wall geometry and its elastic properties. For a given cross-sectional area $A$ it can be expressed as

$$
\mathcal{D}_{A}=\frac{1}{A}\left(\frac{\partial A}{\partial p}\right)_{A},
$$

which according to equation (5) is

$$
\mathcal{D}_{A}=\frac{2 R_{d}}{A} \frac{\partial L(\zeta, p)}{\partial p}=\frac{U_{0} R_{d}^{2}}{A} \frac{\left(1-\sigma^{2}\right)}{E h_{0}} .
$$

Comparison with direct measures of the distensibility $\mathcal{D}_{A}$ confirm the approximate form

$$
c_{A}(x, t)=\frac{1}{\sqrt{\rho_{0} \mathcal{D}_{A}}},
$$

for the wave speed. By using equation (3) and (5) we can finally write the pressure in terms of deformation

$$
p(\zeta, A)=\frac{E h_{0}}{\left(1-\sigma^{2}\right)} \frac{\left(\pi\left(\zeta R_{0}\right)^{2}+A-\zeta R_{0} U_{0}\right)}{U_{0}\left(\zeta R_{0}\right)^{2}} .
$$

The elastic properties for a given $x$-slice of a circular tube are obtained by using estimates for the volume compliance $C_{v o l}$ as suggested in [20], where the empirical approximation in exponential form is

$$
E h_{0}=R_{0}\left(k_{1} \exp \left(k_{2} R_{0}\right)+k_{3}\right)
$$

In these estimates $k_{1}, k_{2}$, and $k_{3}$ are constants. With data for $C_{v o l}$ from Westerhof et al. [32], Stergiopulos et al. [31], and Segers et al. [27] we obtain $k_{1}=2.0 * 10^{6}\left[\frac{\mathrm{kg}}{\mathrm{s}^{2} \mathrm{~m}}\right], k_{2}=-2.253 * 10^{3}\left[\frac{1}{\mathrm{~m}}\right]$, and $k_{3}=8.65 * 10^{4}\left[\frac{\mathrm{kg}}{\mathrm{s}^{2} \mathrm{~m}}\right]$.

\subsection{Governing Equations for Vessel Segments}

The derivation of the governing equations in variables $\mathrm{A}, \mathrm{u}, \mathrm{p}$ can be found in several places $[2,7,11,20,23,28]$ and because they are in common use we will simply state them below without a new derivation. However we will take care of alterations due to our concern - the external wall deformation. Nevertheless we will shortly repeat the main assumptions made. Firstly we assume that blood flow in reasonable large vessels can be modeled as incompressible, Newtonian fluid with constant density $\rho_{0}$ and constant dynamic viscosity $\nu[3,7]$. The Reynolds number $R e=\frac{u d}{\nu}$ is below 2000 in all vessel segments of the cardiovascular system, so that the flow can be assumed to be laminar [7]. The wave velocity may take values as low as $5 \mathrm{~m} / \mathrm{s}$ in the aorta, rising to values around $20 \mathrm{~m} / \mathrm{s}$ in less distensible peripheral arteries or to $35 \mathrm{~m} / \mathrm{s}$ in strongly deformed tubes. However, peak flow velocities are much smaller, generally around $1 \mathrm{~m} / \mathrm{s}$, while they can reach $6 \mathrm{~m} / \mathrm{s}$ in segments of severe deformation. In figure 5 we have plotted the relation for a typical set of $u, c_{A_{d}}, A_{d}$ and $\frac{\partial p}{\partial A_{d}}$ versus the degree of deformation. The fraction of $u / c_{A_{d}}$ for values of $\zeta_{0}=0$ is 0.092 , while for $\zeta_{0}=0.95$ it is 0.16 . Therefore the propagation velocity of the wave can bee seen as large compared to the mean flow velocity of the fluid $(c \gg u)$.

\subsubsection{Conservation of mass and momentum}

The equation of continuity for longitudinal motions is influenced by area changes: Conservation of mass for a control volume $\Omega_{n}$ implies that the mass per unit length $\rho_{0} A$ changes at a rate equal to the efflux $\psi$ minus the gradient of the mass flow rate $\rho_{0} A u$; for impermeable boundaries $\psi=0$. The differential form of the incompressible one-dimensional continuity equation along the $x$-direction is

$$
\frac{\partial A}{\partial t}+\frac{\partial A u}{\partial x}=\psi
$$


(a) Compliance, area, wave and flow velocity versus degree of deformation

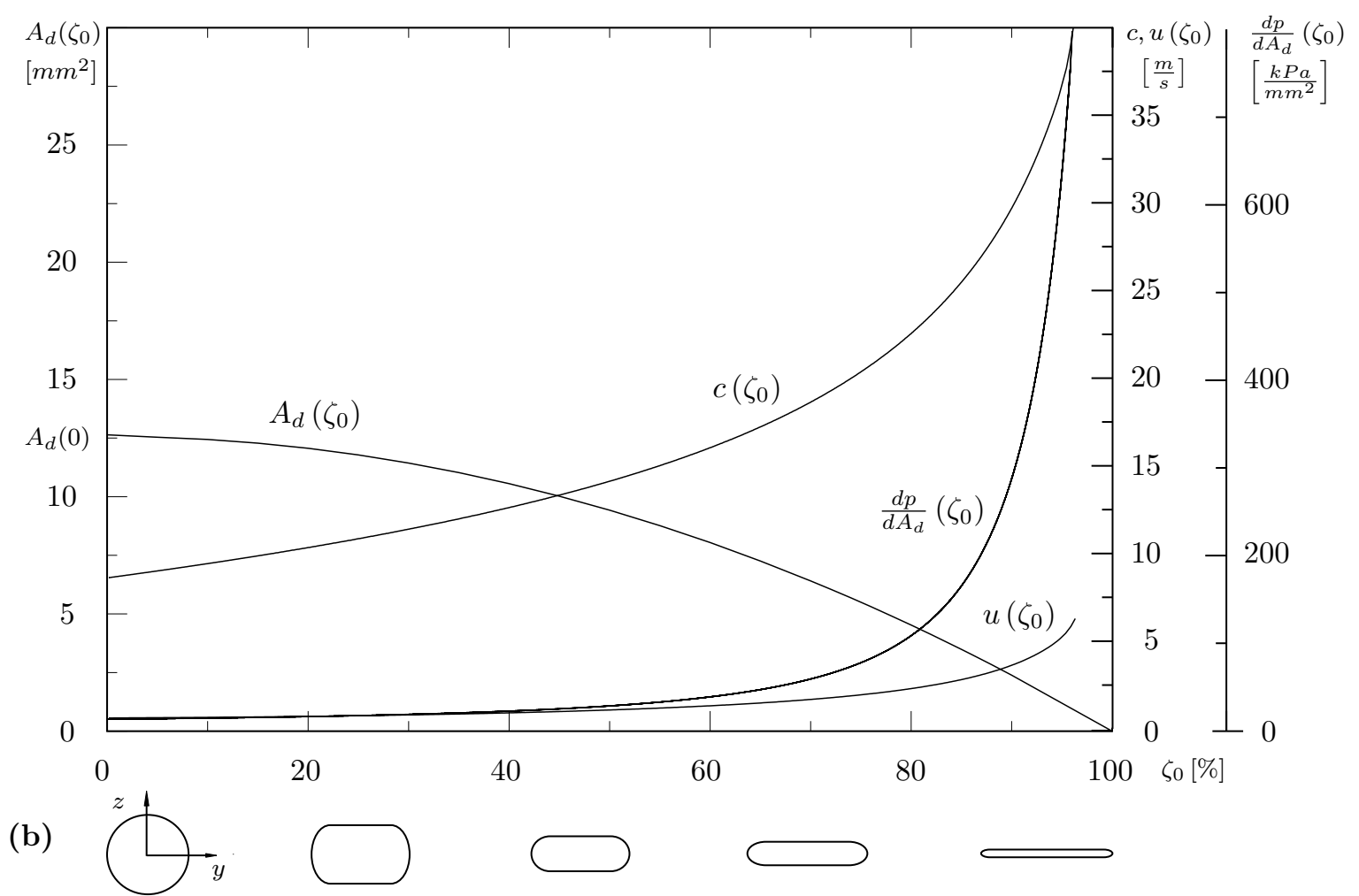

Figure 5. (a) Dependence of compliance, area, wave and flow velocity on deformation $\zeta_{0}$. The gradient $d p / d A_{d}=\frac{1}{\mathcal{D}_{A_{d}} A_{d}}$ is the compliance per unit length. (b) Schematic illustration of the different stages of deformation.

The total cross-sectional area can be written as $A=A^{\prime}+A_{d}$, where $A^{\prime}$ are the perturbations about the equilibrium area $A_{d}$, and consequently the equation (13) is reorganised as

$$
\frac{\partial A^{\prime}}{\partial t}=-\left[\frac{\partial A u}{\partial x}+\frac{\partial A_{d}}{\partial t}\right] .
$$

The derivation of $A_{d}$ with respect to time is a prescribed function depending on $\zeta(x, t)$. It is responsible for the volume displacement caused by the deformation of the tube. However at all points of a particular cross-section the same longitudinal gradient of $p$ is found, and therefore also the same fluid acceleration and hence the same fluid velocity $u$ is found. The most general form of the averaged momentum equation with radial viscous drag is

$$
\frac{\partial A u}{\partial t}+\frac{\partial}{\partial x}\left(\chi A u^{2}\right)+\frac{A}{\rho_{0}} \frac{\partial p}{\partial x}=\mathcal{F}_{\nu} u
$$

where $\chi$ is a momentum correction coefficient and $\mathcal{F}_{\nu} u$ is the viscous friction term. Similar equations are obtained by cross-sectional averaging the cylindrical form of the Navier-Stokes equation. Having satisfied mass and momentum conservation for tubes with external deformation we now adopt a third independent expression for the excess pressure, which was previously derived in equation (11). By the assumption that $E=E(x)$ and 
$A_{d}=A_{d}(x, t)$ and applying the chain rule we obtain the longitudinal pressure gradient

$$
\frac{\partial p}{\partial x}=\frac{\partial p}{\partial A} \frac{\partial A}{\partial x}+\frac{\partial p}{\partial \zeta} \frac{\partial \zeta}{\partial x}+\frac{\partial p}{\partial E} \frac{\partial E}{\partial x} .
$$

So far we have not made any assumptions about the form of the velocity profile. For pulsatile laminar flow in small vessels we assume a parabolic flow profile of the form

$$
\hat{u}=2 \tilde{u}\left(1-\frac{r^{2}}{R^{2}}\right)
$$

Here $\tilde{u}$ is the free stream value of the axial velocity and $R$ is the radius of the total cross-sectional area. Assuming that the radial viscous drag force is perfectly in phase with the mean velocity it can be evaluated by

$$
2 \pi \nu R\left[\frac{\partial \hat{u}}{\partial r}\right]_{R}=-8 \pi \nu u
$$

so that the friction parameter is $\mathcal{F}_{\nu}=-8 \pi \nu$. However the momentum correction coefficient is defined as

$$
\chi(x, t)=\frac{1}{A \tilde{u}^{2}} \int_{S} \hat{u}^{2} d \sigma=\frac{4}{3} .
$$

For reasons that the viscous friction values obtained from the circular orifice do not correspond exactly to those from the noncircular orifices we have assumed that an orifice having a noncircular geometry results in equal friction losses to those obtained from a circular orifice of the same area. Thus any theory from circular orifices can also be extended to situations where noncircular orifices are involved. We note that in the presence of a stenosis the losses are underestimated [31]. This is mainly due to disregarding the losses caused by flow separation at the diverging end of the stenosis $[7,13]$.

\subsubsection{The Characteristic System}

It is convenient to rewrite the set of nonlinear equations for incompressible inviscid fluid flow in elastic tubes with forced deformation of the walls and the pressure-area relationship so that

$$
\begin{aligned}
\frac{\partial A^{\prime}}{\partial t} & =-\left[\frac{\partial A u}{\partial x}+\frac{\partial A_{d}}{\partial t}\right] \\
\frac{\partial u}{\partial t} & =-\left[\frac{u(\chi-1)}{A} \frac{\partial A u}{\partial x}+u \frac{\partial \chi u}{\partial x}+\frac{1}{\rho_{0}} \frac{\partial p}{\partial x}\right]+\frac{\mathcal{F}_{\nu} u}{A} \\
p & =\frac{E h_{0}}{\left(1-\sigma^{2}\right)} \frac{\left(\pi\left(\zeta R_{0}\right)^{2}+A-\zeta R_{0} U_{0}\right)}{U_{0}\left(\zeta R_{0}\right)^{2}} \\
A & =\pi\left(\zeta R_{0}\right)^{2}+2 L(\zeta, p) \zeta R_{0} .
\end{aligned}
$$

These equations cannot be solved analytically and many numerical schemes require the system to be in conservation form. Anyhow a quasi-linear first-order characteristic system for the outgoing $A$ and $u$ variables can be written so that

$$
\frac{\partial \mathbf{U}}{\partial t}+\mathbf{M}(\mathbf{U}) \frac{\partial \mathbf{U}}{\partial x}=\left[\begin{array}{c}
A \\
u
\end{array}\right]_{t}+\left[\begin{array}{cc}
u & A \\
\frac{1}{\rho_{0} \mathcal{D}_{A} A} & u
\end{array}\right]\left[\begin{array}{l}
A \\
u
\end{array}\right]_{x}=\left[\begin{array}{l}
0 \\
f
\end{array}\right],
$$

whereas $f$ is the forcing term

$$
f=-\frac{1}{\rho_{0}}\left(\frac{\partial p}{\partial \zeta} \frac{\partial \zeta}{\partial x}+\frac{\partial p}{\partial E} \frac{\partial E}{\partial x}\right)+\frac{\mathcal{F}_{\nu} u}{A}
$$


We note that the gradients $\frac{\partial E}{\partial x}$ and $\frac{\partial \zeta}{\partial x}$ are zero on the boundary. However the eigenvectors of $\mathbf{M}(\mathbf{U})$ are

$$
\lambda(\mathbf{M})=\left[\begin{array}{c}
u+\frac{1}{\sqrt{\rho_{0} \mathcal{D}_{A}}} \\
u-\frac{1}{\sqrt{\rho_{0} \mathcal{D}_{A}}}
\end{array}\right], \quad \mathbf{L}(\mathbf{M})=\left[\begin{array}{cc}
A \sqrt{\rho_{0} \mathcal{D}_{A}} & -A \sqrt{\rho_{0} \mathcal{D}_{A}} \\
1 & 1
\end{array}\right]=\left[\begin{array}{cc}
\frac{\partial w^{+}}{\partial A} & \frac{\partial w^{-}}{\partial A} \\
\frac{\partial w^{+}}{\partial u} & \frac{\partial w^{-}}{\partial u}
\end{array}\right],
$$

and $\mathbf{M}=\mathbf{L} \mathbf{\Lambda} \mathbf{L}^{-\mathbf{1}}$ with

$$
\mathbf{\Lambda}(\mathbf{M})=\left[\begin{array}{cc}
\lambda^{+} & 0 \\
0 & \lambda^{-}
\end{array}\right]
$$

the diagonal eigenvalue matrix. We approximate the outgoing characteristic variables in absence of viscous forces i.e. $\mathcal{F}_{\nu}=0$ so that we can write the following set of decoupled scalar equations

$$
\frac{\partial w^{ \pm}}{\partial t}+\Lambda \frac{\partial w^{ \pm}}{\partial x}=0
$$

The wave speed of the nonlinear system $c_{A}=\frac{1}{\sqrt{\rho_{0} \mathcal{D}_{A}}}$ is always positive and generally much larger than the velocity of blood. The characteristics of the system have opposite directions which is indicated by the \pm signs. The characteristic variables are found by using the eigenmatrix $\mathbf{L}(\mathbf{M})$ such that $\frac{\partial w^{ \pm}}{\partial u}=1$ and $\frac{\partial w^{ \pm}}{\partial A}= \pm A \sqrt{\rho_{0} \mathcal{D}_{A}}$

$$
w^{ \pm}(x, t)=u \pm \int_{A_{d}}^{A} \frac{1}{A \sqrt{\rho_{0} \mathcal{D}_{A}}} d A=u \pm 2 \frac{\sqrt{\rho_{0} \mathcal{D}_{A_{d}}}-\sqrt{\rho_{0} \mathcal{D}_{A}}}{\sqrt{\rho_{0} \mathcal{D}_{A_{d}}} \sqrt{\rho_{0} \mathcal{D}_{A}}}=u \pm 2\left(c_{A}-c_{A_{d}}\right) .
$$

The characteristic variables given in equation (29) are also Riemann invariants of the nonlinear system. Provided that the perturbations $A^{\prime}$ are small a system of linearised equations can be written. The distensibility for the equilibrium cross-sectional area $A_{d}$ is

$$
\mathcal{D}_{A_{d}}=\frac{2 R_{d}}{A_{d}} \frac{\partial L(\zeta, p)}{\partial p}=\frac{U_{0} R_{d}^{2}}{A_{d}} \frac{\left(1-\sigma^{2}\right)}{E h_{0}}=\frac{2 R_{0} \zeta}{(2-\zeta)} \frac{\left(1-\sigma^{2}\right)}{E h_{0}} .
$$

The pressure-area relationship in equation (11) reduces to

$$
p\left(\zeta, A^{\prime}\right)=\frac{E h_{0}}{\left(1-\sigma^{2}\right)} \frac{A^{\prime}}{U_{0}\left(\zeta R_{0}\right)^{2}}
$$

and the linearised system can be written as

$$
\frac{\partial \mathbf{U}_{d}}{\partial t}+\mathbf{M}_{d}\left(\mathbf{U}_{d}\right) \frac{\partial \mathbf{U}_{d}}{\partial x}=\left[\begin{array}{c}
A^{\prime} \\
u
\end{array}\right]_{t}+\left[\begin{array}{cc}
0 & A_{d} \\
\frac{1}{\rho_{0} \mathcal{D}_{A_{d}} A_{d}} & 0
\end{array}\right]\left[\begin{array}{c}
A \\
u
\end{array}\right]_{x}=\left[\begin{array}{l}
0 \\
f
\end{array}\right]
$$

with the following eigenvectors of $\mathbf{M}_{d}\left(\mathbf{U}_{d}\right)$

$$
\lambda_{d}\left(\mathbf{M}_{d}\right)=\left[\begin{array}{c}
\frac{1}{\sqrt{\rho_{0} \mathcal{D}_{A_{d}}}} \\
-\frac{1}{\sqrt{\rho_{0} \mathcal{D}_{A_{d}}}}
\end{array}\right], \quad \mathbf{L}_{d}\left(\mathbf{M}_{d}\right)=\left[\begin{array}{cc}
A_{d} \sqrt{\rho_{0} \mathcal{D}_{A_{d}}} & -A_{d} \sqrt{\rho_{0} \mathcal{D}_{A_{d}}} \\
1 & 1
\end{array}\right]=\left[\begin{array}{cc}
\frac{\partial w^{+}}{\partial A_{d}} & \frac{\partial w^{-}}{\partial A_{d}} \\
\frac{\partial w^{+}}{\partial u} & \frac{\partial w^{-}}{\partial u}
\end{array}\right] .
$$

The diagonal matrix of eigenvalues in (27) and the scalar equations in (28) are identical for the linear system, anyhow the characteristic variables are

$$
w^{ \pm}(x, t)=u \pm \int_{A_{d}}^{A} \frac{1}{A_{d} \sqrt{\rho_{0} \mathcal{D}_{A_{d}}}} d A=u \pm \frac{A^{\prime}}{A_{d} \sqrt{\rho_{0} \mathcal{D}_{A_{d}}}}=u \pm c_{A_{d}} \frac{A^{\prime}}{A_{d}}
$$


where $c_{A_{d}}=\frac{1}{\sqrt{\rho_{0} \mathcal{D}_{A_{d}}}}$ is the wave speed for the linearised system. Both systems are hyperbolic and subcritical so that we require one boundary condition at each end of the tube.

\section{INTERFACE AND BOUNDARY CONDITIONS}

\subsection{In- and Outflow Boundary Conditions}

The boundary condition at the inflow to the arterial tree is applied to the aorta, and the outflow boundary conditions are applied to all peripheral terminals of the network. They are both imposed through the characteristic system. The inflow boundary condition is given by the flow velocity, the area, or a relation between them. The shape of the pulse wave in the ascending aorta however is generated by the inflow from the aortic valve, so that we represent the inflow either by a periodic extension of a measured flow wave or in exponential form as given in [22].

$$
q_{\text {in }}(t)=q_{0} \frac{t}{\tau^{2}} \exp ^{\frac{-t^{2}}{2 \tau^{2}}}
$$

The inflow amplitude of the exponential waveform is $q_{0}$, while $\tau$ is its attack time. To incorporate the boundary conditions for the linear system we make use of the following relations.

$$
A^{\prime}=\frac{A_{d}}{c_{A_{d}}} \frac{\left(w^{+}-w^{-}\right)}{2}, \quad u=\frac{\left(w^{+}+w^{-}\right)}{2}
$$

Different types of outflow conditions my be applied. Non-reflecting boundary conditions or perfectly matched layers (PML) are applied by the use of one-way wave equations, which for the forward and backward travelling characteristics are given in (37) and (38) respectively.

$$
\begin{aligned}
\left(\frac{\partial}{\partial x}+\frac{1}{c} \frac{\partial}{\partial t}\right) w^{+}\left(x_{\max }, t+\delta t\right) & =\left(\frac{\partial}{\partial x}+\frac{1}{c} \frac{\partial}{\partial t}\right) w^{+}\left(x_{\max }-\delta x, t\right) \\
\left(\frac{\partial}{\partial x}-\frac{1}{c} \frac{\partial}{\partial t}\right) w^{-}\left(x_{\min }, t+\delta t\right) & =\left(\frac{\partial}{\partial x}-\frac{1}{c} \frac{\partial}{\partial t}\right) w^{-}\left(x_{\min }+\delta x, t\right)
\end{aligned}
$$

This however assumes that the primary wave direction is normal to the boundary. There are several ways to account for peripheral reflections at the terminals starting from pure resistive load, where the outflow is proportional to the pressure over three and four-element windkessel models $[18,30]$ to a structured tree outflow condition suggested in [22].

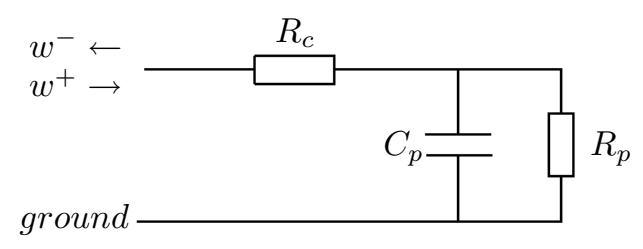

FiguRE 6. Three-element windkessel analog circuit.

We have chosen to use a reasonable three-element windkessel model (WK) given in [34]. The main advantage of this model is to consider the compliant-capacitive effects due to microvessels and arterioles. The lumped analog electrical circuit is shown in figure 6. According to [29] the differential equation in the time domain satisfied by the circuit is

$$
\frac{\partial p}{\partial t}=R_{c} \frac{\partial q}{\partial t}-\frac{p}{R_{p} C_{p}}+\frac{q\left(R_{p}+R_{c}\right)}{R_{p} C_{p}}
$$


with $R_{p}$ and $C_{p}$ being the peripheral resistance and compliance respectively. However $R_{c}$ is the characteristic impedance of the terminating vessel, which for large vessels is a real number and modelled by a resistor.

The total peripheral resistance $R_{t}=R_{p}+R_{c}$ for each of the terminals was estimated by the total arterial peripheral resistance and the distribution of flow through the various branches [31]. The ratio $R_{c} / R_{t}$ was estimated in [24] by fit to data and found to be approximately 0.2 . Finally, the arterial compliance $C_{p}$ for each segment was estimated from the total volume compliance [33]. Parameter values for $R_{t}$ and $C_{p}$ can be found in $[19,31]$.

\subsection{Interface Conditions}

Finally, we need three conditions at each of the bifurcations to close the system of equations. Physically motivated by the conservation of mass and momentum through the bifurcation, the mass flux balance results in $q_{1}=q_{2}+q_{3}$ while the momentum flux results in the continuity of total pressure $p_{t}$. The interface conditions for the nonlinear system are therefore

$$
\begin{aligned}
A_{p} u_{1} & =A_{d_{1}} u_{d_{1}}+A_{d_{2}} u_{d_{2}}, \\
p_{p}+\frac{1}{2} \rho_{0} u_{p}^{2} & =p_{d_{1}}+\frac{1}{2} \rho_{0} u_{d_{2}}^{2}, \\
p_{p}+\frac{1}{2} \rho_{0} u_{p}^{2} & =p_{d_{2}}+\frac{1}{2} \rho_{0} u_{d_{2}}^{2}
\end{aligned}
$$

while the linear system has similar conditions except that the static pressure is continuous across the bifurcation. The subscripts $p, d_{1}$ and $d_{2}$ stand for the parent, $1^{\text {st }}$ and $2^{\text {nd }}$ daughter vessels respectively. Anyway to apply the interface conditions we approximate the characteristic variables $w^{+}$and $w^{-}$at the boundary using the one way wave equations (37) and (38) for the inflow and outflow respectively.

\section{Application}

\subsection{Myocardial Bridge}

Based on 83 angiographies, Dodge et al. $[8,9]$ presented a normal anatomic distribution of coronary artery segments and proposed a terminology, which we used for our model of the left coronary artery (LCA): the left main coronary artery (LMCA) bifurcates into the left anterior descending artery (LAD) and the left circumflex artery (LCxA). The main branches of the LAD include the $1^{\text {st }}, 2^{\text {nd }}$ and $3^{\text {rd }}$ diagonal branch (D1, D2, D3) and the $1^{\text {st }}, 2^{\text {nd }}$ and $3^{\text {rd }}$ septal branch (S1, S2, S3). The main branches of the LCxA include the $1^{\text {st }}$ and $2^{\text {nd }}$ obtuse marginal branches (OM1, OM2). The exact intrathoratic location and course of each one of the 27 arterial segments and branches of the LCA are illustrated in figure 7 (a), while the main bifurcation and a separated myocardial bridge are shown in (b) and (c) respectively.

The lumen diameter of the LMCA orifice measured $4.5 \mathrm{~mm}$, while the corresponding values of the LAD and LCxA were $3.7 \mathrm{~mm}$ and $3.4 \mathrm{~mm}$, respectively. The outlet diameter of the LAD at the apex of the heart was $0.9 \mathrm{~mm}$, while the corresponding diameter of the LCxA at the outlet was $1.3 \mathrm{~mm}$. For the first, second and third diagonal the corresponding diameters were $1.1 \mathrm{~mm}, 1.0 \mathrm{~mm}$ and $0.9 \mathrm{~mm}$, respectively, while for the first, second and third septal diameters were $0.9 \mathrm{~mm}, 0.7 \mathrm{~mm}$ and $0.7 \mathrm{~mm}$, respectively. For the LCxA branches, the outlet diameters of the first and second obtuse marginal were of $1.1 \mathrm{~mm}$ and $1.0 \mathrm{~mm}$.

\section{Results}

In the following we determine the influence of the myocardial bridge on the total volume flux, which presumably depends on the values of the terminal resistance, the heart rate, the phase and degree of the deformation function and finally the friction term within the bridge. We have chosen the parameters for the simulation accordingly: 
(a)

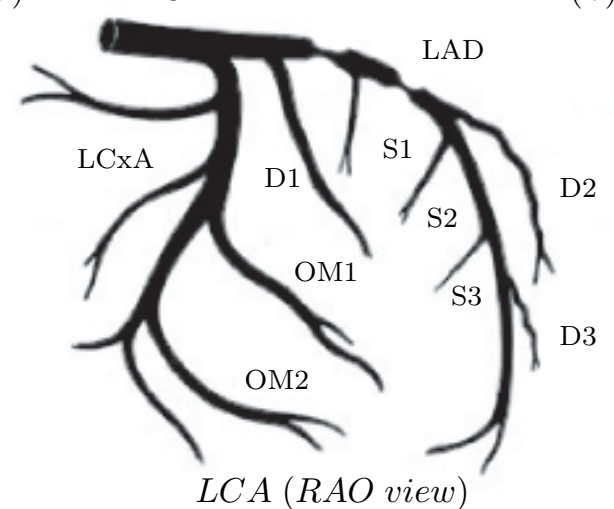

(b)

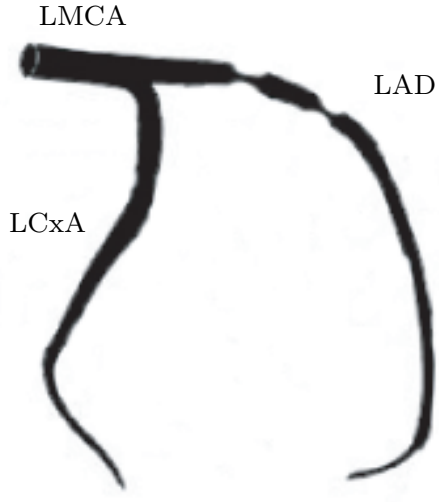

Bifurcation (c)

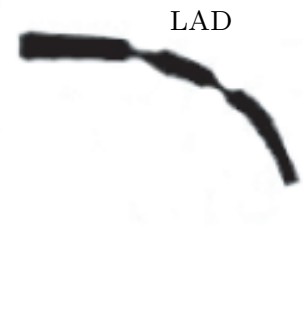

Segment

FiguRE 7. The simulation domains for three simplified topologies. (a) The 27 main segments of the LCA, (b) the main bifurcation and (c) the separated myocardial bridge.

As previously mentioned the mid LAD is divided into equally spaced bridge segments with length $10 \mathrm{~mm}$. We applied an exponential flow wave with amplitude $q_{0}=400 \mathrm{~cm}^{3} / \mathrm{s}$ and attack time of $\tau=0.14 \mathrm{~s}$ to the aorta, resulting in a total cardiac output $(\mathrm{CO})$ of $5.25 \mathrm{l} / \mathrm{min}$. The time dependent part of the deformation function along the $z$-axis $f_{z}(t)$ was assumed to be periodic (see measurements in [16]) and is approximated by $f_{z}(t)=\sum_{n=1}^{3} \frac{0.8}{n} \sin \left(n \omega(t+\triangle t)+\phi_{n}\right)$. Here $\triangle t$ is the time shift with respect to the cardiac cycle and the phases $\phi_{n}$ in radian were chosen to be $\phi_{1}=3.5, \phi_{2}=1.5$, and $\phi_{3}=3.9$. Finally the deformation curvature is described by the transition length $l_{t}=2 \mathrm{~mm}$ and the degree of vessel deformation $\zeta_{0}=0.95$, which is equivalent to a reduction in lumen area by $90 \%$. We have chosen the spatial accuracy to resolve the curvature of deformation $(\delta x=4$ grid points per $m m)$ and the time step width to satisfy the CFL condition $(\delta t=10 \mu s)$.

The results are based on a network topology mentioned in $[10,15,22,28]$ and the topology of the major 48 coronary arteries presented by Dodge et al.. The LCA with two myocardial bridges in series is shown in figure 7 (a). Two simplified cases were investigated by removing distal and proximal segments leaving the main bifurcation (b) and a separated myocardial bridge (c). The input flow waveform to the segment in (c) was taken from a previous simulation of the reference LCA, directly proximal to the myocardial bridge, while the input to the bifurcation in (b) was equal to (a), but reduced by $6.7 \%$ to match the flow proximal to the bridge. The peripheral resistance's for the bifurcation and the separated segment were chosen by adapting the peripheral resistance's of the main LAD and LCxA branches, so that the flow rate in the reference LAD and LCxA were equal in all three cases. The results comparing the left coronary arterial tree (a) and the simplified
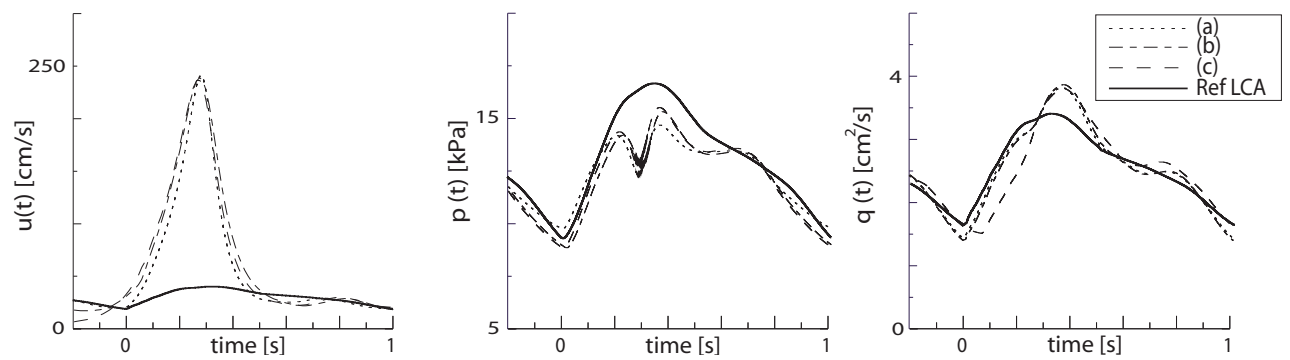

Figure 8. The flow velocity, pressure and volume flow of the myocardial bridge in setup (a), (b) and (c) are plotted versus time. The degree of deformation was $\zeta_{0}=0.95$. The thick solid line indicates the reference of a normal LAD.

domains (b), (c) illustrated in figure 8, show good qualitative agreement, so we decided to further investigate the parameter variation only for the LCA. 
(I) severity
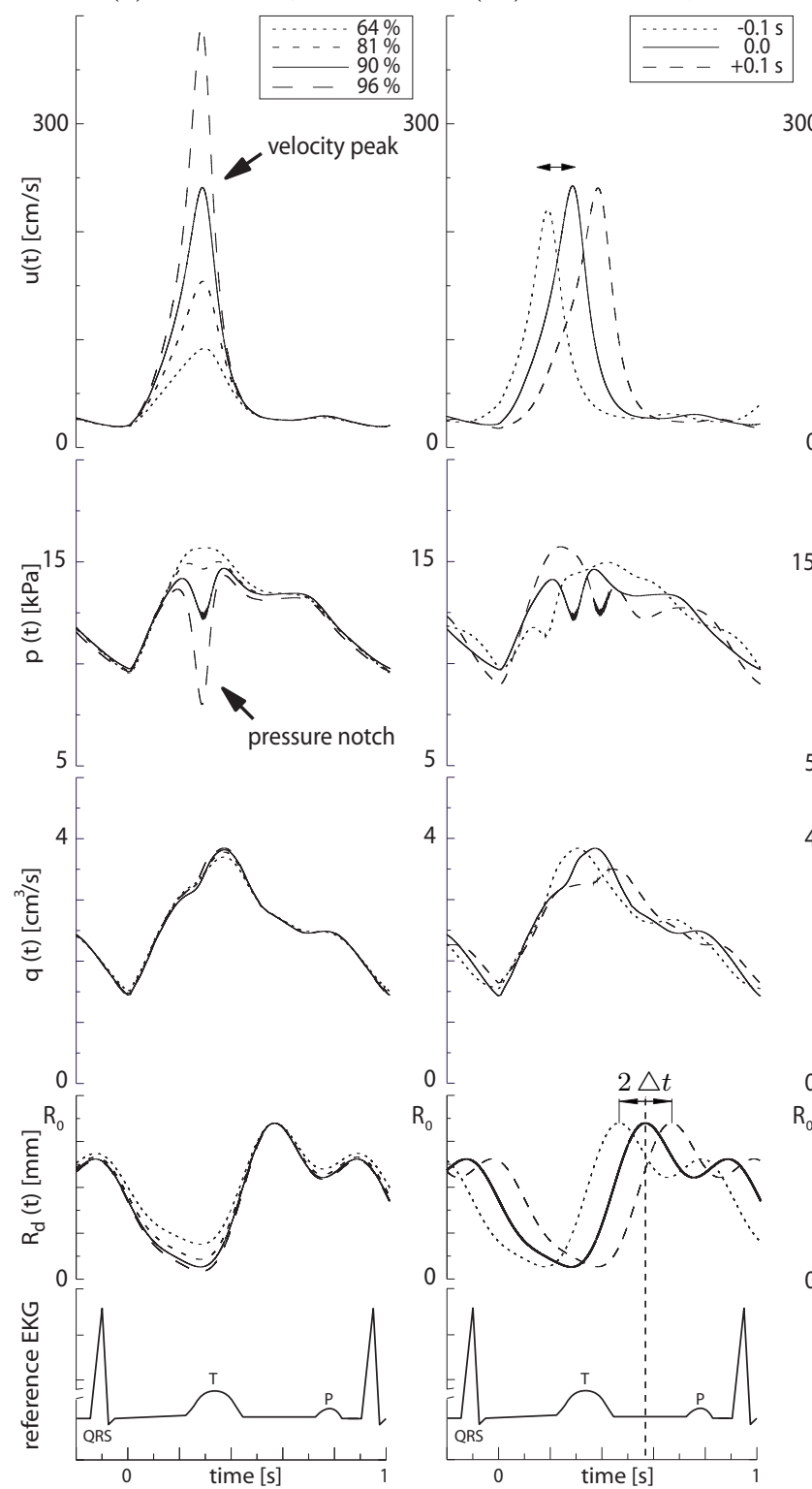

(II) time shift
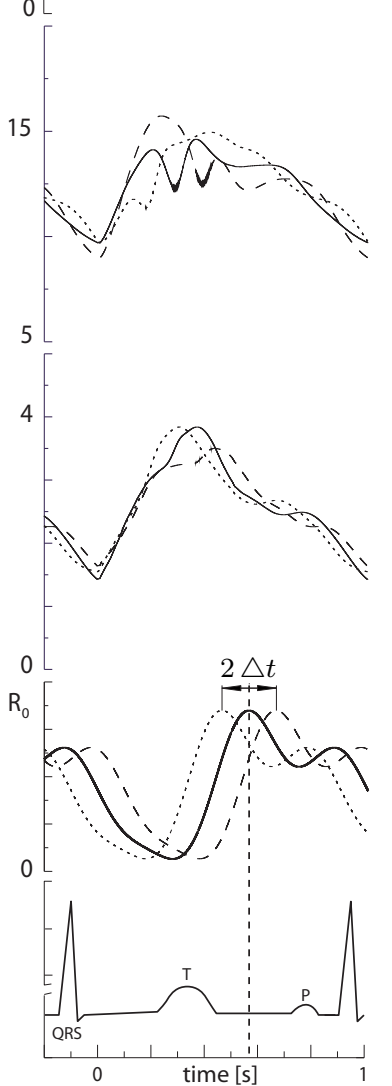

(III)

length

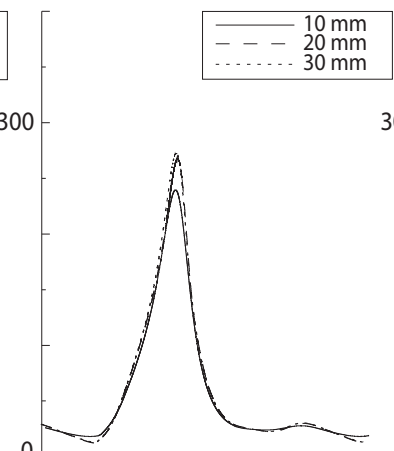

(IV) termination
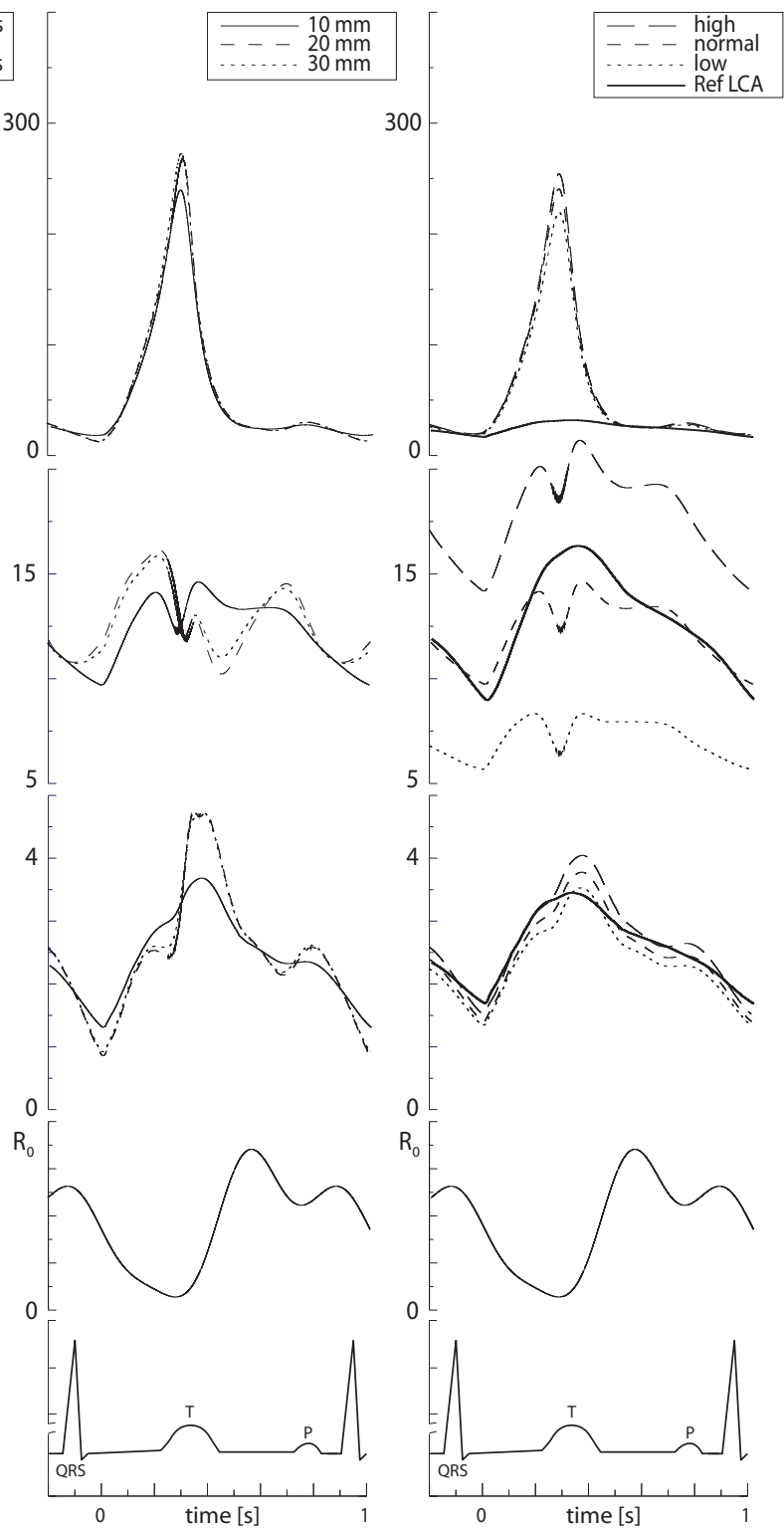

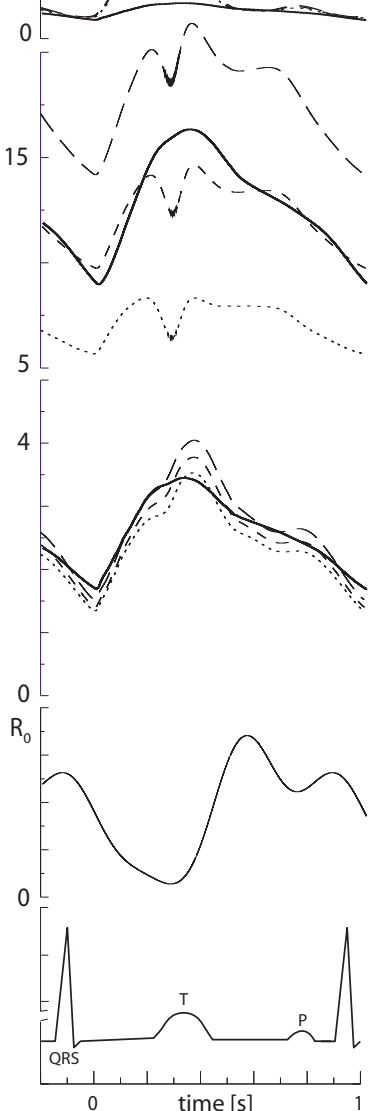

Figure 9. The flow velocity, pressure, volume flow and deformation of the myocardial bridge are plotted in relation to a reference EKG. Relaxation following asymmetric compression is delayed into diastole. The reference of a normal LAD (thick solid line in (IV)) is compared to the values taken within the myocardial bridge (dashed lines). In (I) we compare bridges of different severity. During deformation the peak flow velocity is increased (velocity peak) and in the nonlinear case a pressure notch is found. (II) illustrates the effect of time shifting the deformation with respect to the cardiac cycle. Further we change the length of the stenosis in (III) and the peripheral resistance in (IV).

The flow velocity, pressure, volume flow and deformation of the myocardial bridge are plotted in relation to a reference EKG (figure 9). The thick solid line in (IV) indicates the reference of a normal LAD having 
peak flow velocities of $30 \mathrm{~cm} / \mathrm{s}$, remaining dashed lines are values taken within the myocardial bridge. We note that the linear equations are only valid for deformations $<50 \%$, because the flow velocity is small in that case $\left(u_{s 2, s 4}<1 \mathrm{~m} / \mathrm{s}\right)$. However for stronger lumen reduction the flow velocity is significantly increased during deformation and hence the nonlinear term becomes more pronounced. This becomes evident in the characteristic pattern of flow velocity (velocity peak) and pressure (pressure notch) in figure 9 (I). The depth of the pressure notch increases with flow velocity and is therefore not persistent during phases of small deformation. Compared to the pressure of the normal LAD the depth of the notch is of the order $1.5 k P a \leq \triangle p \leq 8 k P a$ for area reductions between $81 \%$ and $96 \%$. For higher deformations $(>98 \%)$ the depth of the notch exceeds the pressure inside the tube, i.e. negative pressure is observed which would generally cause a collapse of the tube. We note that the present model is only valid if the pressure remains positive, which is satisfied for deformations smaller $98 \%$.

A pressure-flow wave typically requires $20 \mathrm{~ms}$ to travel the distance between the aortic valve and the myocardial bridge. In contrast the deformation of the vessel during systole happens instantaneously. The pressure in the left ventricle however has to overcome the aortic pressure to allow wave ejection, typically this time-span is $80 \mathrm{~ms}$. The phase of deformation with respect to the wave entering the muscle bridge is therefore dependent on the relative distance to the aortic valve and the time required by blood compression in the left ventricle. This however strongly depends on anatomy, so that we investigate the pressure-flow patterns for deformations shifted in time by $\Delta t= \pm 0.1 \mathrm{~s}$ (figure 9 (II)). Another reason to investigate time shifted deformations is that large amount of blood volume is transferred during diastole. The characteristic pattern of flow acceleration and deceleration (velocity peak and pressure notch) change their position with deformation phase. The maximum peak flow velocities are found if the deformation has opposite phase with respect to the flow wave.

In (III) the segment length of the myocardial bridge was modified. On the basis of anatomic relevant values we used $10 \mathrm{~mm}, 20 \mathrm{~mm}$ and $30 \mathrm{~mm}$. The peak flow velocity increases with segment length and the volume flow is significantly increased during relaxation (suction) $\left(\triangle q \approx 1 \mathrm{~cm}^{3} / \mathrm{s}\right)$.

Finally in figure 9 (IV) we have shown the influence of peripheral resistance on the mean pressure. The variation was observed in three categories $\left(\triangle R_{t}= \pm 30 \%\right)$ for low, normal and high peripheral resistance. The peak flow velocity, volume flow and mean pressure increase with peripheral resistance.

Published data from patient studies and experiments in $[14,16,17]$ show good qualitative and quantitative agreement to our simulations. A similar set of parameters was used to compare the peak flow velocities which in our simulations are $u_{s 1}=24.2 \mathrm{~cm} / \mathrm{s}$ proximal, $u_{s 2, s 4}=86 \mathrm{~cm} / \mathrm{s}$ within, and $u_{s 5}=26.5 \mathrm{~cm} / \mathrm{s}$ distal the bridge. Accordingly the values from in vitro measurements are $u_{s 1}=28.6 \pm 8.8 \mathrm{~cm} / \mathrm{s}, u_{s 2, s 4}=63.7 \pm 26.2 \mathrm{~cm} / \mathrm{s}$, and $u_{s 5}=24.7 \pm 14.4 \mathrm{~cm} / \mathrm{s}$. We found a total volume flux of $135.6 \mathrm{ml} / \mathrm{min}$ and $135.9 \mathrm{ml} / \mathrm{min}$ for the normal and the dynamic LAD respectively, i.e. a limiting effect on the volume flow was not observed. Anyhow we found that the peak values of the excess pressure are almost constant throughout the bridge, which is not consistent with the findings in [14], where a high pressure chamber in the centred segment $\left(\Omega_{3}\right)$ was observed. We suppose that this is only observed for total occlusion or collapse of the bridge.

\section{Discussion and Conclusion}

We have presented a method for simulation of blood flow through forced deformation of vessel segments. The application to myocardial bridges shows good quantitative agreement to peak flow velocities observed in in vitro measurements [14]. We could also confirm the velocity peak found in qualitative analysis of the Doppler flow profiles within the myocardial bridge, which is characterised by an abrupt early diastolic flow acceleration, a rapid mid-diastolic deceleration, and a mid-to-late-diastolic plateau [16]. The acceleration of the fluid is caused due to rapid reduction of the cross-sectional area of the vessel during the contraction phase, while a rapid deceleration is caused during relaxation. By comparison to static stenosis we found that the anomalous accelerations are dependent on the phase and degree of the deformation function. Maximum peak flow velocities are observed for deformation gradients having opposite phase with respect to the fluid acceleration caused by the pressure gradient, i.e. for maximum deformation during the systole. 
However during deformation secondary flow is caused by momentum transfer between solid and fluid. The amount of fluid displaced depends on the normal diameter and length of the bridge, and the degree of the deformation. In the case that this additional fluid motion exceeds the normal flow, reverse flow at the proximal end of the bridge is observed during inward motion of the wall, while the like for the distal end is observed during relaxation.

We found that the total perfusion to the myocardium is strongly dependent on the severity of the muscle bridge. For weak severity with lumen area reduction $(<65 \%)$ the pressure and volume flow are approximately constant throughout the bridge and hence the influence of the bridge on the total perfusion to the myocardium is small. However vessel compression with area reductions $>90 \%$ show reasonable pressure gradients across the bridge during the deformation maximum. This can be explained by the circumstance that the viscous friction and convective term are influenced by the flow velocity, which rapidly increases with deformation. The linear term due to viscous friction is dominant for small deformations, while the nonlinear term due to flow separation is dominant for elevated flow during strong deformation. This is consistent with the findings in [13], where the terms accounted for $65 \%$ and $35 \%$ at resting coronary flow and for $33 \%$ and $67 \%$ at peak coronary flow respectively. In contrast to static stenosis these losses are not persistent during periods of small deformation, so that the pressure distal the bridge recovers during this time span. Consequently the pressure drop and flow reduction across static stenosis are more pronounced than in myocardial bridges with equal severity. However we suggest that a critical myocardial bridge should be defined in terms of its effect during maximal flow rather than resting flow.

In this work we have presented a theoretical basis to compute flow and pressure dynamics in a model of myocardial bridges. We believe that the parameters and equations in this article are detailed enough to describe the physiological consequences also in a clinical setting, however this remains to be confirmed by in vivo studies. The functional consequence, especially for severe systolic compression, is consistent with clinical findings published in the literature $[14,16,17]$, where myocardial bridging is found to be responsible for myocardial ischaemia. The comparison of our findings with the published data from patient studies supports a potential clinical relevance of our simulation.

\section{REFERENCES}

[1] P. Angelini, M. Tivellato, and J. Donis. Myocardial bridges: a review. Prog Cardiovasc Dis., 26:75-88, 1983.

[2] D. H. Bergel. Cardiovascular Fluid Dynamics I. Academic Press London, 1972.

[3] D. H. Bergel. Cardiovascular Fluid Dynamics II. Academic Press London, 1972.

[4] J.P. Broser. Simulation of fluid flow in blood vessels. Master's thesis, Universität Heidelberg, 2002.

[5] C. G. Caro, T. J. Pedley, R. C. Schroter, and W. A. Seed. The mechanics of the circulation. Oxford University Press, 1978.

[6] Landau L. D. and Lifschitz E. M. Hydrodynamik. Harri Deutsch Verlag, 1990.

[7] U. Dinnar. Cardiovascular Fluid Dynamics. CRC Press Inc., 1981.

[8] J. T. Dodge, B. G. Brown, E. L. Bolson, and H. T. Dodge. Intrathoracic spatial location of specified coronary segments of the normal human heart. Circulation, 78(5):1167-1180, 1988.

[9] J. T. Dodge, B. G. Brown, E. L. Bolson, and H. T. Dodge. Lumen diameter of normal human coronary arteries. Circulation, 86(1):232-246, 1992.

[10] L. Formaggia, D. Lamponi, and M. Tuveri. Numerical modelling of $1 \mathrm{~d}$ arterial networks coupled with lumped parameters description of the heart. 2000.

[11] Y. C. Fung. Biomechanics Mechanical Properties of Living Tissues. Springer Verlag, Heidelberg, 1981.

[12] Y. C. Fung. Biodynamics Circulation. Springer Verlag New York, 1984.

[13] K. L. Gould. Quantification of coronary artery stenosis in vivo. Circulation Research, 57:341-353, 1985.

[14] H.G. Klues and E.R. Schwarz. Disturbed intracoronary hemodynamics in myocardial bridging. Circulation, 96(9):2905-2913, 1997.

[15] Keshaw Kumar. Anatomy of the human coronary arterial pulsation. J. Anat. Soc. India, 52(1):24-27, 2003.

[16] S. Möhlenkamp, H. Eggebrecht, T. Ebralidze et al. Muskelbrücken der Koronararterien: mögliche ischämierelevante Normvarianten. Herz, 30(1):37-47, 2005.

[17] S. Möhlenkamp, W. Hort, J. Ge, and R. Erbel. Update on myocardial bridging. Circulation, 106(20):2616-2622, 2002.

[18] A. Noordergraaf. Circulatory System Dynamics. Academic Press London, 1978.

[19] M. S. Olufsen. Modeling the arterial System with Reference to an Anesthesia Simulator. PhD thesis, IMFUFA Roskilde University, 1998. 
[20] M. S. Olufsen. Structured tree outflow condition for blood flow in larger systemic arteries. American Physiological Society, 1999.

[21] M. S. Olufsen, A. Nadim, and L. A. Lipsitz. Dynamics of cerebral blood flow regulation explained using a lumped parameter model. Am. J. Physiol. Regulatory Integrative Comp Physiol, 282:R611-R622, 2002.

[22] M. S. Olufsen, C. S. Peskin, and J. Larsen. Numerical simulation and experimental validation of blood flow in arteries with structured-tree outflow conditions. Annals of Biomedical Engineering, 28:1281-1299, 2000.

[23] T. J. Pedley. The fluid dynamics of large blood vessels. Cambridge University Press, 1980.

[24] J. Raines, M. Jaffrin, and A. Shapiro. A computer simulation of arterial dynamics in the human leg. J Biomech, 7:77-91, 1974.

[25] H. C. Reyman. Disertatio de vasis cordis propriis. PhD thesis, Med Diss Univ Göttingen., 1737.

[26] A. K. Roger. An introduction to the mathematical theory of waves. American Mathematical Society, 3 edition, 2000.

[27] P. Segers, F. Dubois, D. Wachter, and P. Verdonck. Role and relevancy of a cardiovascular simulator. J. Cardiovasc. Eng., (3):48-56, 1998

[28] S. J. Sherwin, V. Franke, and J. Peiro. One-dimensional modelling of a vascular network in space-time variables. Journal of Engineering Mathemetics, 47:217-250, 2003.

[29] N. Stergiopulos, J. J. Meister, and N. Westerhof. Evaluation of methods for estimation of total arterial compliance. American Physiological Society, pages 1540-1548, 1995.

[30] N. Stergiopulos, B. E. Westerhof, and N. Westerhof. Total arterial inertance as the fourth element of the windkessel model. American Physiological Society, 1999.

[31] N. Stergiopulos, D. F. Young, and T. R. Rogge. Computer simulation of arterial flow with applications to arterial and aortic stenosis. J. Biomech., 25:1477-1488, 1992.

[32] N. Westerhof, Bosman, C. J. DeVries, and A. Noordergraaf. Pressure and flow in the systemic arterial system. J. Biomech., 5:629-641, 1972.

[33] N. Westerhof, F. Bosman, C. J. De Vries, and A. Noordergraaf. Analog studies of the human systemic arterial tree. J. Biomech., 2:121-143, 1969.

[34] N. Westerhof, G. Elzinga, and P. Sipkema. An artificial arterial system for pumping herarts. J. Appl. Physiol., 31:776-781, 1971.

[35] M. Zamir. The Physics of Pulsatile Flow. Biological Physics Series. Springer-Verlag Heidelberg, 2000. 